\title{
Nouveau type de stars, nouveaux actes de langage? La construction de l'ethos discursif de youtubeurs français à travers les rituels d'ouverture et de clôture de leurs vidéos
}

\author{
New Type of Stars, New Speech Acts? \\ The Construction of the Discursive Ethos of French YouTubers \\ Throughout the Opening and Closing Rituals of Their Videos
}

Fanni Filyó

Université Eötvös Loránd de Budapest, Hongrie

\begin{abstract}
Résumé : Cet article s'articule autour de la notion de l'ethos des youtubeurs célèbres de France et vise à mettre en lumière la construction identitaire qui se réalise dans les actes de langage ouvrant et clôturant leur communication. La nature de ces actes dans la scène générique de YouTube nécessite une interrogation sur leur fonction rituelle, d'une part, et une analyse du stéréotypage des stratégies, d'autre part. Ces points de vue permettent de comprendre le mécanisme langagier des figures emblématiques des chaînes YouTube françaises ainsi que la relation interpersonnelle établie par leur activité.
\end{abstract}

Mots-clés : YouTube, ethos, identité discursive, analyse du discours, actes de langage.

Abstract: This article focuses on the notion of ethos of famous YouTubers from France and aims to highlight the construction of identity that takes place in the speech acts opening and closing their communication. The nature of these acts on YouTube's generic scene requires, on the one hand, to question their ritualistic function and, on the other hand, an analysis of the stereotyping of strategies. These points of view allow us to understand the language mechanism of the emblematic figures of French YouTube channels as well as the interpersonal relationship established by their activity.

Keywords: YouTube, ethos, discursive identity, discourse analysis, speech acts.

\section{Introduction}

Selon la définition d'Aristote (cit. d'après Maingueneau, 2002, p. 1), la construction de l'ethos, réalisée essentiellement par l'élaboration de l'énonciation, " consiste à faire bonne impression [...], à donner une image de soi capable de convaincre ")'. L'acte de persuader est, en effet, en lien étroit avec l'activité des youtubeurs : construisant un ethos acceptable par le public prototypique, les vidéastes gagnent davantage

I En réalité, la notion s'avère plus hybride : il s'agit, selon Maingueneau (2002, p. 7), d'un « comportement socialement évalué, qui ne peut être appréhendé hors d'une situation de communication précise, intégrée elle-même dans une conjoncture socio-historique déterminée ». 
d'adhésion. Dans ce procédé, les actes de langage ouvrant et clôturant les discours jouent un rôle crucial surtout en raison de leur fonction d'accroche (Combe-Celik, 2014). Bien que les actes de langage prennent une fonction rituelle et deviennent des stratégies quasi-conventionnelles ${ }^{2}$, leur réalisation dans une situation de communication concrète contribue à la construction de l'ethos discursif individuel. Sans prétendre à l'exhaustivité, le présent travail vise à mettre en lumière des stratégies de ce processus par le biais des actes de langage qui ouvrent la communication et de ceux qui la clôturent, y compris le remerciement. Pour ce faire, nous avons étudié l'activité des youtubeurs les plus connus de France: Squeezie, Cyprien et Natoo.

\section{Constitution du corpus}

Malgré la facilité d'accès aux énoncés, à cause du volume important des données sur le web et d'une interaction constamment ouverte, nous nous retrouvons face à la problématique de la représentativité (Pierozak, 2011, pp. 16-22). Dans notre cas, la représentativité n'est pas assurée par la notion de genre mais par celle du succès des youtubeurs faisant l'objet du présent travail. Cela nous offre un corpus construit de manière ad hoc, contenant des énoncés qui permettent une analyse discursive hypothétique "en concomitance avec [l]es corpus ouverts et la description des données linguistiques " (Rakotonoelina, 2011, p. 38). À cet égard, il est important de souligner que notre travail se base sur une analyse qualitative, ce qui a pour conséquent que les constatations ainsi que la constitution du corpus s'avèrent, dans une certaine mesure, subjectives. Ceci ayant pour objectif de discerner les possibilités de réalisation des stratégies discursives dans la perspective d'effectuer une analyse quantitative.

Ainsi, après avoir consulté des listes ${ }^{3}$ de classement des youtubeurs français ayant le plus d'abonnés, nous avons sélectionné dans notre corpus des vidéos de Squeezie, de Cyprien (les deux premiers youtubeurs de la liste) et de Natoo (la première parmi les femmes), ces vidéos datant de l'ouverture de leur chaîne jusqu'en 2020. Une étude intégrale et générale de leurs productions nous a permis de choisir à peu près 15 vidéos de chacun des youtubeurs afin de constituer un corpus pour l'analyse détaillée des actes de langage. Pour ce faire, nous avons pris en considération les critères de parution afin de dépister l'évolution des vidéastes, ensuite le sujet de la vidéo afin d'avoir des sujets similaires pour chaque youtubeur, et finalement le type de vidéo afin de pouvoir distinguer les mécanismes discursifs selon les contraintes liées au genre ou à un certain type de production.

\footnotetext{
2 L'image de soi, véhiculée par des stratégies discursives, « est conditionnée par des cadres sociaux et institutionnels préexistants dans la logique desquels elle s'inscrit " (Amossy, 2010, p. 37). La scène générique de YouTube semble aujourd'hui fournir des modèles prédisposés aux vidéastes qui y prennent parole.

3 https://socialblade.com/youtube/top/country/fr/mostsubscribed (12/07/2020). La liste devra être reconstituée de manière dynamique car elle change relativement vite. Ainsi, au niveau du nombre d'abonnés, quoique Cyprien figure parmi les premiers, il semble diminuer la production des podcasts. L'étude peut tout de même être révélatrice du point de vue des mécanismes langagiers et peut donner de nouvelles pistes d'interrogation ou de comparaison avec les stratégies discursives des nouveaux arrivants sur YouTube.
} 
Tableau 1 - Le corpus ${ }^{4}$

\begin{tabular}{|c|c|c|c|c|}
\hline \multirow{2}{*}{ Youtubeur } & \multirow{2}{*}{ Vidéo } & \multicolumn{2}{|c|}{ Longueur } & \multirow{2}{*}{ Code } \\
\hline & & Vidéo & Au total & \\
\hline \multirow{14}{*}{ Squeezie } & Video commentée : Crysis 2 & 08:32 & \multirow{17}{*}{ 04:25:52 } & SJ11 \\
\hline & $\begin{array}{l}\text { DÉSOLÉ, IL N'Y A PAS DE JEU. (There is no } \\
\text { game) }\end{array}$ & $07: 24$ & & SJI5 \\
\hline & SQUEEZIE - ÊTRE SEUL CHEZ SOI & 03:43 & & SP12 \\
\hline & LE FLÉAU DE YOUTUBE & 05:47 & & SP16 \\
\hline & $\begin{array}{l}\text { VOUS N'ALLEZ PAS FERMER L'OEIL DE LA } \\
\text { NUIT... }\end{array}$ & $11: 37$ & & SP18 \\
\hline & QUI ÉCRIT CES ARTICLES ET POURQUOI? ? & 13:29 & & SP19 \\
\hline & Une vidéo... Surprise! & 04:55 & & SAl1 \\
\hline & $\begin{array}{l}150.000 \text { abonnés: Mon formidable } \\
\text { parcours, grâce à vous. }\end{array}$ & $06: 30$ & & SA 12 \\
\hline & MERCI & 07:39 & & SA14a \\
\hline & MON CADEAU DE NOËL POUR VOUS! & 03:35 & & SA $14 \mathrm{~b}$ \\
\hline & LES VILAINS COMMENTAIRES... & 06:16 & & SA15 \\
\hline & MES BIDES... & $06: 28$ & & SA16 \\
\hline & JE SORS UN LIVRE! & 04:16 & & SA17 \\
\hline & Un message sincère & 07:37 & & SA 18 \\
\hline \multirow{3}{*}{ Cyprien } & Le vélo en ville - Cyprien & 03:24 & & CP1la \\
\hline & La canicule (3D) - Cyprien & 03:14 & & CP11b \\
\hline & Les boss - Cyprien & 05:03 & & CPllc \\
\hline
\end{tabular}

\footnotetext{
${ }^{4}$ Les titres figurant dans ce tableau ainsi que tous les extraits (transcription des vidéos, commentaires) cités dans ce travail sont reproduits tels qu'ils apparaissent sur YouTube, sans aucune correction.
} 


\begin{tabular}{|c|c|c|c|}
\hline & Cyprien - La Wii U & $03: 36$ & CP12 \\
\hline & Cyprien - Le mariage homosexuel & $04: 25$ & CP13a \\
\hline & Cyprien - L'école & $04: 49$ & CP13b \\
\hline & CYPRIEN - SNAPCHAT & $04: 30$ & CP14a \\
\hline & Cyprien - Les jeunes et la technologie & $04: 26$ & CP14b \\
\hline & CYPRIEN - LE DESSIN & $05: 23$ & CP16 \\
\hline & CYPRIEN - REGARDER DES SÉRIES & $05: 15$ & CP17 \\
\hline & APPRENDRE UNE LANGUE - CYPRIEN & $06: 14$ & CP18 \\
\hline & Cyprien - 3 millions d'abonnés! & $01: 39$ & CA13 \\
\hline & CYPRIEN - MON ANNÉE 2014 & $07: 19$ & CA14 \\
\hline & CYPRIEN - MA PREMIÈRE BD ! & $05: 19$ & CA15a \\
\hline & MON POTE HOLLANDE - Cyprien répond 4 & $06: 32$ & CA15b \\
\hline & JE REGARDE MES PREMIÈRES VIDÉOS... & $12: 02$ & CA19 \\
\hline \multirow{10}{*}{ Natoo } & La maison de campagne - Natoo & $04: 31$ & NP11 \\
\hline & Les gens sales - Natoo & $06: 04$ & NP12 \\
\hline & Les Mamans - Natoo & 05:08 & NP13 \\
\hline & Avoir 2 chiens - Natoo & $05: 38$ & NP15 \\
\hline & Les femmes sur YouTube - Natoo & $05: 14$ & NP16 \\
\hline & CELIB' ACHAT - Natoo & $10: 35$ & NP18 \\
\hline & LA VIEILLESSE - Natoo & $08: 30$ & NP20 \\
\hline & FAQ Snapchat - Natoo & $06: 27$ & NA15a \\
\hline & Ecrire un livre - "Icônne by Natoo" & $04: 18$ & NA15b \\
\hline & $\begin{array}{l}\text { Dédicaces d'lcônne et pluie de cadeaux! } \\
\text { - Natoo }\end{array}$ & $04: 50$ & NA15c \\
\hline
\end{tabular}




\begin{tabular}{|l|l|l|l|l|}
\hline \multirow{2}{*}{$\begin{array}{l}\text { Joyau Magique by Natoo (ma marque de } \\
\text { bijoux!) }\end{array}$} & $03: 38$ & \multirow{3}{*}{ NA16 } \\
\cline { 1 - 3 } & 5M D'ABONNÉS : MA PIRE JOURNÉE !!! & $20: 01$ & & NA20 \\
\hline
\end{tabular}

Une précision concernant la typicité et l'emploi notionnel s'impose, notamment en raison de la définition vague du podcast. À l'origine, " un podcast est un contenu audio numérique que l'on peut écouter n'importe où, n'importe quand, grâce à la technologie du flux RSS $\|^{5}$. YouTube offre la possibilité d'un enregistrement vidéo des podcasts, ce qui marque une évolution du genre : c'est l'apparition du vidéo podcast

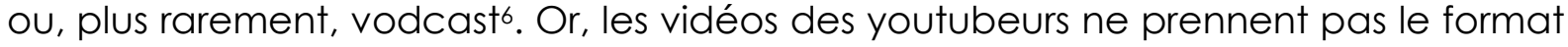
mp4 comme un simple support mais elles s'appuient dessus: le visuel assure l'intentionnalité réussie du discours. Néanmoins, dans le langage courant ainsi que dans celui des vidéastes, le terme « podcasts " prévaut. Ainsi, au sens large, à part les gameplays ${ }^{7}$, pratiquement toutes les vidéos traitées tomberaient dans la catégorie podcast. Dans cette perspective, dans le cas de Cyprien et de Natoo, nous utilisons podcast lorsque le contenu est basé sur des sketchs (apparaissant, dans la plupart des cas, sous forme de jeux de rôles) et dans le cas de Squeezie, les podcasts sont des vidéos se concentrant également sur un sujet précis lié à l'espace virtuel (essentiellement réalisées sur support de l'espace virtuel), malgré le fait qu'il refuse d'être classé comme tel. En ce qui concerne les vidéos catégorisées comme « autres » dans notre corpus, elles privilégient plutôt des synthèses, que ce soit celle d'une période (année de carrière), de la réalisation de soi (publications, remerciement aux visionneurs) ou d'une interaction engagée à l'égard des visionneurs (poser des questions).

\section{Actes d'ouverture}

L'acte de salutation prête au youtubeur un ethos "disposé à engager avec le destinataire un échange communicatif » (Kerbrat-Orecchioni, 2016, p. 111), celui-ci devient ainsi binaire. Or, la chaîne de la communication implique une asymétrie dans l'interaction sur YouTube, par conséquent, la fonction de l'acte d'ouverture diffère. Contrairement aux situations de communication directes, la fonction d'accroche est bien prononcée dans les discours sur YouTube : «que ce soit par l'image ou par le texte les youtubeurs veillent à établir un contact avec leurs destinataires dès l'ouverture de la vidéo " (Combe Celik, 2014, p. 275). Capter l'attention du public est important du point de vue perlocutoire ${ }^{8}$ et persuasif, de plus, il convient d'ajouter que les rituels s'empreignent souvent d'originalité et deviennent des traits distinctifs. Le visionneur, quant à lui, se contente de se retrouver face à une ouverture

\footnotetext{
5 https://podmust.com/podcast-definition-histoire/ (21/12/2020).

${ }^{6}$ https://www.buzzsprout.com/blog/podcasting-on-youtube (21/12/2020).

7 Gameplayers sont des producteurs de contenu amateurs qui enregistrent des épisodes de jeu de vidéos, les complétant éventuellement de commentaires ou d'effets spéciaux (visuels ou sonores), pour les partager ensuite en ligne (Glózer \& Guld, 2015, p. 41). Nous appelons ces types de vidéos «jeux commentés $1 \%$.

8 Selon Kerbrat-Orecchioni (2014, p. 22), il s'agit d'un « acte effectué par le fait de dire quelque chose ». Cela peut se réaliser de manière explicite, mais c'est également le cas lorsque l'énonciation possède des intentions cachées.
} 
accoutumée: la reconnaissance le réjouit d'autant plus qu'elle lui fait sentir de la complicité.

\subsection{Ouverture comme identité discursive}

L'exemple de Squeezie révèle l'importance du trait distinctif des actes d'ouverture, puisque, en effet, il garde pendant relativement longtemps (jusqu'en 2016) l'acte de présentation dans sa salutation, malgré le fait que son nom soit connu : Yo tout le monde, c'est Squeezie, on se retrouve pour une nouvelle vidéo. Le passage à d'autres formes (dont Et bien bonjour mes amis) informe sur la (re)configuration de la relation interpersonnelle, motivée par l'arrivée à maturité du youtubeur et par la proximité ressentie à l'égard de sa communauté. Ce sentiment est renforcé par l'adresse affective mes amis, laquelle contribue à établir une relation parasociale?. Nous constatons donc que la relation interpersonnelle est en lien étroit avec la construction de l'ethos : si l'identité du youtubeur se construit, au début de sa carrière, au sein de sa communauté (on se retrouve), plus tard, elle se réalise en se détachant textuellement de la communauté. Conformément à l'évolution de l'identité discursive, la finalité de la communication change: Squeezie attribue moins d'importance à la stabilisation de son identité.

Cyprien et Natoo, quant à eux, créent, dès le début, un ethos détaché des visionneurs par le biais des salutations telles que Bonjour, c'est Cyprien, il y a quelques jours [...] ou Bonjour, je vais vous parler / vous allez regarder [...] de la part de Natoo. Pour ce qui est de Cyprien, il a d'abord recours à la présentation de soi qu'il abandonne rapidement, c'est-à-dire, dès qu'il obtient la reconnaissance suffisante pour établir un ethos de quasi-vedette. II ne vise pas, comme Squeezie, à maintenir une relation affective avec son public, mais se concentre davantage sur les possibilités issues du genre du podcast, dont celle de l'omission de la salutation en tant que telle. En ce qui concerne Natoo, la fonction rituelle est pratiquement la seule à caractériser sa salutation, ainsi l'intention d'établir une proximité avec le public s'avère secondaire, d'autant plus que l'ouverture pose explicitement les rôles liés au genre du podcast. Même si, à un certain moment, nous observons la volonté d'originalité par l'énoncé Hey, hey, hey, le retour à l'ouverture évoquée ci-dessus suppose un ethos plutôt indépendant : Natoo se montre moins soucieuse d'établir une image plus polie que nécessaire, elle semble faire ses sketchs selon son gré.

II faut également souligner que certains youtubeurs, en particulier Squeezie, semblent renoncer au caractère itératif et donc distinctif de ces actes. Nous supposons que le youtubeur est à la recherche d'un nouveau type de démarcation, c'est-à-dire qu'il souhaite s'écarter des formules prototypisées sur YouTube et, par conséquent, abandonner la salutation comme trait distinctif. Les discours sur YouTube

\footnotetext{
9 YouTube crée une situation de communication particulière entre les youtubeurs et les visionneurs. Elle s'avère être plus hiérarchisée que celle des réseaux sociaux (Filyó, 2021, p. 92), tout en engendrant un sentiment de proximité entre les interlocuteurs, surtout de la part des visionneurs. L'auto-exposition des youtubeurs témoigne ainsi d'une asymétrie propre à la relation parasociale. Cette notion désigne le fait que la célébrité est effectivement intégrée dans un réseau de conversations dont, pourtant, le caractère est unilatéral (Marshall, 2010,38) dans la mesure où le visionneur n'a pas la possibilité d'engager une conversation avec le youtubeur comme personnes privées. L'unilatéralité ne veut guère dire le manque d'interaction, car un grand nombre de réactions ultérieures émergent face au discours du vidéaste, entraînant ainsi une influence potentielle sur l'activité discursive.
} 
témoignent d'un changement dynamique au sein duquel se trouve la nécessité de se renouveler pour concurrencer les nouveaux arrivants sur la plateforme.

Grâce au cadre discursif, l'absence de l'acte de salutation devient un effet de style, comme le montre l'exemple suivant: Il y a quelques jours, j'étais invité au mariage de ma cousine (CP13a). L'in medias res peut, en effet, servir de fonction d'accroche, prenant le destinataire par surprise. En plus, ces actes mettent souvent le je au centre de l'attention, renforçant ainsi la relation asymétrique entre le youtubeur et son public. En témoignent également les ouvertures qui recourent à l'apostrophe comme la suivante : vous savez, j'adore le Japon (CP18). Cyprien établit une image prototypique de ses visionneurs selon laquelle ils suivent ses vidéos et retiennent ses centres d'intérêt (puisque cet énoncé fait référence à son propre discours préalablement posté). De cette manière, le visionneur est placé à la fois dans une position de connivence et d'idolâtrie, maintenant ainsi une relation parasociale.

Natoo, elle, se filme, sans aucune réserve, dans son entourage intime, comme l'illustre l'un de ses énoncés d'ouverture : Bonjour, aujourd'hui, je vais faire la vidéo à partir de mon lit parce que c'est le seul endroit où c'est pas le bordel dans mon appart (NP12). Cette phrase témoigne, tout de même, d'une prise en considération des visionneurs comme complices, ainsi ils peuvent ressentir une certaine proximité. À part cela, il lui arrive également d'interpeller les visionneurs comme dans l'énoncé suivant: Bonjour, aujourd'hui je voudrais vous faire prendre le bonheur frais de la campagne en vous emmenant chez ma mère et chez mon beau-père (NP11). L'exemple décrit une proximité physique, le visionneur accède aux scènes familiales privées. De plus, Natoo filme souvent avec et pour sa mère (NP13), cette dernière devient également une personne médiatisée. Une fois de plus, la relation parasociale est amplifiée.

Lorsque nous comparons les stratégies, nous constatons que le travail soigneux s'avère plus apte à éveiller l'attention des visionneurs, d'autant plus que Cyprien, recourant à l'humour, implique le visionneur dans le processus, ce qui entraîne une connivence plus forte que ce que nous pouvons remarquer dans les phrases d'accroche à fonction phatique de Natoo. Ainsi, quoiqu'il ait pour fonction d'établir une distance émotionnelle, l'acte humoristique est plus "personnel " que l'acte phatique. Néanmoins, lorsqu'il s'agit d'apostropher le public, les énoncés de Natoo instaurent plus de proximité affective que ceux de Cyprien, justement en raison de l'effet de distanciation émotionnelle de l'humour. Squeezie, qui accorde une grande importance à la recherche identitaire, construit l'ethos d'un youtubeur " ami des abonnés ॥, ce qui entretient la relation parasociale.

\subsection{Mise en public de soi}

Comme évoqué ci-dessus, la particularité des discours des youtubeurs réside dans le fait de créer une proximité, tout en se plaçant au centre d'attention. Dans la suite, nous esquissons trois types d'énoncés apparaissant en début de discours, qui révèlent explicitement la mise en public de soi.

Les énoncés tels que Moi, je déteste, Moi, ce que j'adore, etc. ouvrent la vidéo par une exposition explicite du moi, suivis souvent d'une expression de sentiment, de point de vue, d'habitude, etc. L'emploi du pronom personnel tonique suppose une prise de parole et une prise de position, ce qui renvoie à une interaction. Le fait d'évoquer un point de vue personnel par moi permet de rebondir sur un sujet, le pronom devient donc 
un élément de cohésion et de cohérence. Un autre type d'introduction est constitué par l'énoncé suivant: Bonjour, je vais vous parler. Si, dans le premier cas, la présence d'un « je » imposait, de manière implicite, la présence d'un « tu », dans le présent cas, le youtubeur positionne les visionneurs derrière un écran sur lequel il apparaît comme sujet parlant, donc protagoniste explicité. Ce rituel d'ouverture semble être plus fréquent lorsque le vidéaste présente un projet, et non pas lorsqu'il s'agit d'un podcast. Ce type d'énoncé révèle une relation amicale dans la mesure où les partenaires partagent des propos personnels et s'écoutent, mais de manière asymétrique : c'est la relation parasociale à la manière de YouTube.

Enfin, l'ouverture du type Comment ça va ? moi... mérite une étude plus détaillée. L'acte a théoriquement deux utilisations : celle phatique, qui initie une conversation et celle d'une question prise littéralement, qui s'intéresse à l'état d'esprit du destinataire. Cette « duplicité illocutoire " (Kerbrat-Orecchioni, 2014, p. 112) devient un avantage pour le youtubeur grâce à la scène générique qui lui permet d'insérer les deux fonctions dans un même acte. Vu que la question arrive en tout début de conversation, elle s'interprète comme un acte phatique, une expansion à la salutation ${ }^{10}$. Cet acte contribue à créer une face positive, puisqu'il " permet au questionneur, de faire preuve d'une sollicitude polie [...] et au questionné d'avoir l'agréable impression que sa personne est l'objet d'une certaine considération, sans être pour autant tenu de se livrer ॥ (Kerbrat-Orecchioni, 2014, p. 116). C'est de cette manière que la question est identifiée de la part du visionneur qui n'a, en effet, pas la possibilité d'y répondre. Néanmoins, cette question exige une réaction : soit une réponse type préféré lorsqu'il s'agit d'un acte orienté, donc plutôt phatique, soit une réponse type non préféré lorsque l'acte est identifié comme une vraie question et on a le désir de raconter ses malheurs (Kerbrat-Orecchioni, 2014, p. 115). Quoi qu'il en soit, l'énonciateur peut imaginer une réponse de la part du visionneur dans le cas de l'utilisation phatique du terme: une réponse positive (dont la valeur de vérité n'a aucune importance car élément phatique, Bartha, 1998, p. 37) ou la répétition de la question par politesse et par rituel. Si la question imaginée est interprétée au sens littéral par le youtubeur, il se met à parler de son état d'âme ou physique mais, contrairement à la constatation de Kerbrat-Orecchioni, il s'agit aussi des émotions positives et négatives. Le glissement de la question rituelle à la vraie question est toujours une stratégie du questionneur (Kerbrat-Orecchioni, 2014, p. 119), c'est ainsi que la conversation commence avec des informations vraies (Bartha, 1998, p. 37). Stratégie apparue chez tous les youtubeurs, elle assure ensuite une cohésion et une cohérence à leur discours tout en y impliquant le destinataire. Ici, nous remarquons qu'au-delà du postulat selon lequel "un 'je' implique automatiquement un 'tu' " (Amossy, 2010, p. 105) », la présence d'un «tu » permet également la mise en scène d'un «je».

\section{Actes de clôture}

Le rituel de la clôture de la communication sur YouTube diffère considérablement de celui de la situation de communication directe. Certes, dans le sens strict, dire au revoir (à bientôt, ciao, etc.) est un acte pleinement phatique, mais l'acte de prendre congé ne s'épuise pas uniquement dans les rituels phatiques: il se complète, voire se transforme en acte perlocutoire de persuasion. L'objectif des youtubeurs, « tout en

10 D'après Kerbrat-Orecchioni (2014, p. 113), « [p]lus il vient tôt, plus il ressemble à une salutation ». 
marquant leur originalité est de tisser des liens avec ceux qui visionnent leurs vidéos en une certaine forme de prosélytisme " (Combe Celik, 2014, p. 279). Dans cette perspective, deux actes de langage se retrouvent liés l'un à l'autre : la persuasion et le remerciement.

\subsection{Acte de persuasion}

L'acte de persuasion vise à démultiplier les abonnés, les partages, les interactions, en bref, la notoriété. Le pathos, à savoir l'argument qui touche les émotions de l'allocutaire (Amossy, 2012, p. 209), devient alors l'élément dominant. En effet, les youtubeurs lient souvent le contenu de la vidéo à l'appartenance potentielle du destinataire au même groupe que le vidéaste, cette stratégie s'appuyant sur un processus identificatoire : Toi aussi tu regardes des séries? Mais c'est trop génial ! Alors tu peux partager ma vidéo sur Facebook et rejoindre ma page! (CP17). Bien que la valeur de vérité de l'argument soit médiocrell, l'effet escompté est assuré par le pathos et par l'humour qui se cache dans cet acte : l'identification au youtubeur est facilement déclenchable, car la frontière de la personne publique et privée s'efface (Glózer \& Guld, 2015, p. 47). Le dispositif légitime, en effet, la mise en public de la vie privée et c'est cette authenticité qui est valorisée par le public (Gallant, 2017, p. 3) et qui devient l'objet d'admiration, non pas le talent exceptionnel comme chez les vedettes classiques (Glózer \& Guld, 2015, pp. 34-35). Cet aspect donne l'impression que le sujet parlant est «l'un des nôtres» (Glózer \& Guld, 2015, p. 38), ce qui est souvent appuyé dans les discours, aussi bien de la part des vidéastes (on a grandi ensemble, SA14b), que des visionneurs (L'histoire de ma vie, CP13b). Si c'est l'un des siens, le sujet parlant offre également une image du public, qui voudra effectivement se reconnaître dans l'ethos montré (Amossy, 2012, p. 79), la persuasion peut ainsi s'effectuer (Maingueneau, 2002, pp. 2-3).

Nous constatons également que le pronom tu à référent multiple ${ }^{12}$ est régulièrement employé dans ces actes. Son but est de viser l'individu, le gagnant ainsi de manière plus efficace. Le discours de Natoo en témoigne :

Donc mon message c'est 'Lance-toi'. Si t'es une meuf qui hésite à lancer sa chaîne d'humour, de philo, de gaming, littérature, science, cinéma, bah, n'hésite pas, mets de côté le jugement des autres et fais-le pour toi.

La proximité établie grâce à l'apparition du tu sert d'outil d'accentuation et rend l'acte de persuasion plus efficace. En outre, le référent multiple, mais restreint, du tu s'oppose à un type d'individus, ainsi l'emploi contextuel du pronom rassemble le groupe des femmes résistant à la stigmatisation. En effet, Natoo définit sa propre activité au sein d'un médium empreint des stéréotypes de la société hors-ligne, et se met dans la position du porte-parole d'un nous « non-dit ॥ (Amossy, 2010, p. 156) ${ }^{13}$. II en découle que l'énoncé contribue à établir non seulement l'ethos de la youtubeuse,

\footnotetext{
${ }^{11}$ Ce type d'argumentation peut être identifié comme une fausse causalité du paralogisme. Celui-ci est psychologiquement persuasif mais logiquement incorrect (Copi \& Burgess Jackson, 1996 cité dans Amossy, 2012, p. 171).

12 Par pronom tu à référent multiple, nous entendons l'ensemble des visionneurs, imagé comme un seul interlocuteur.

13 II ne reste pas tout au long implicite, car peu après l'énoncé présenté, la youtubeuse explicite le groupe de nous dont elle est représentative par l'intermédiaire d'une marque d'intertextualité : We want you.
} 
mais il démasque également le processus du retravail de l'ethos prédiscursif, stéréotypé.

Contrairement aux exemples précédents, dans le discours de Squeezie, le passage au tu ciblant l'individu ne possède pas forcément un caractère perlocutoire explicite. Son énoncé récurrent toi qui regardes cette vidéo est généré essentiellement par le sentiment de proximité, celui-ci, pour autant, vise à convaincre, puis faire agir le visionneur.

Ensuite, l'humour s'avère également une stratégie recherchée, qu'il soit basé sur la polyphonie : si [...] tu l'as aimée, tu peux l'aimer-c'est logique ça-tu peux t'abonner à ma chaîne, SP12 ; sur le face work ${ }^{14}$ : Abonne-toi ! Enfin si t'as envie... tu fais comme tu veux. J'ai dit ça comme ça, CP14a; ou sur la menace ludique :

Et voilà, vous connaissez un peu mieux cette petite créature qui partage ma vie à présent et j'espère que vous l'appréciez parce que sinon [écrit et image: coup de bourrelet]. N'hésite pas à mettre un pouce bleu et à t'abonner, merci et vive les chiens (NP15).

Le visionneur n'est pas seulement interpellé mais également impliqué dans le décodage ${ }^{15}$ du message dont l'informativité élevée (l'information inattendue) déstabilise la communication mais rend la persuasion plus efficace (Beaugrande \& Dressler, 2000, p. 32). L'acte humoristique agit donc sur les émotions par le sentiment de joie, tout en créant, cependant, une distanciation au niveau affectif.

Finalement, nous constatons le même caractère distinctif des actes de clôture que dans le cas de ceux d'ouverture. Squeezie accentue non seulement l'importance de la clôture, l'énoncé ciao tout le monde, peace devient le trait distinctif le plus marquant dont l'abandon crée de la déception parmi les visionneurs. Parallèlement à sa phrase d'accroche, la clôture on se retrouve pour une prochaine vidéo renforce l'appartenance au même groupe que les visionneurs, de plus, elle annonce la continuation de leur activité commune, devenue habitude. En ce qui concerne Cyprien, il ne se crée pas vraiment de phrase emblématique de son identité discursive, mais se concentre plutôt sur le travail de ses actes de persuasion basés sur l'humour ou l'effet de surprise. Quant à Natoo, la prise de congé du type je vous embrasse, au revoir témoigne de la proximité (je vous embrasse), certes, mais le caractère ritualisé y est codé et, de ce fait, la proximité ne cède ni la place à l'affectivité comme dans le cas de Squeezie, ni à l'implication particulière du visionneur comme chez Cyprien.

\subsection{Acte de remerciement}

L'acte de remerciement relève également, de manière indirecte, de la persuasion (via le pathos). De plus, comme moyen d'agir sur les émotions de la communauté, l'acte de remerciement, apparaissant à la fin de la vidéo, peut être lié, au niveau textuel, à celui de persuasion (Merci d'avoir regardé cette vidéo et si vous l'avez aimée, n'hésitez pas à la liker et à vous abonner, je vous embrasse, au revoir, NP20). L'argument de pathos entre pleinement en jeu ici, car l'énoncé transmet au public le

\footnotetext{
14 Le face work (" travail de figuration » ou « ménagement des faces » en français) est une notion établie par Goffman, qui consiste à élaborer des actes qui ne font perdre la face à aucun des participants de I'interaction (Amossy, 2012, p. 206 ; Kerbrat-Orecchioni, 2014, p. 72).

15 Selon Charaudeau (2006, pp. 22, 27), l'activité du décodage permet à l'acte humoristique de rendre l'énonciateur complice, ce jeu énonciatif s'appuie donc sur un calcul de l'interlocuteur entre le dit et le non-dit.
} 
rôle de bienfaiteur. C'est ainsi que l'énoncé peut être interprété comme un acte perlocutoire, donc les visionneurs agissent: ils (dis)likent, partagent la vidéo, commentent ou s'abonnent à la chaîne.

En effet, le remerciement est un acte de langage rituel; régi par le face work, il s'effectue selon le principe d'équilibre, afin d'annuler une dette (Kerbrat-Orecchioni, 2014, pp. 122-135): celle du soutien des abonnés. Nous observons ainsi une construction, voire une réinvention de l'ethos, qui va à l'encontre de l'asymétrie causée par la position de quasi-vedette. Cette stratégie devient un modèle pour l'ethos "conventionnalisé » des youtubeurs (c'est éventuellement explicité, comme l'illustre la phrase de Natoo : j'ai décidé de rééquilibrer les choses en passant la pire journée possible, NA20). Nous nous demandons toutefois si cet acte de langage a vraiment pour objectif principal de rééquilibrer la relation interpersonnelle. Certes, la politesse que cet acte manifeste accorde la préférence à «l'alter sur l'ego » (KerbratOrecchioni, 2014, p. 139), mais c'est une politesse abondante servant la force perlocutoire, dont témoigne l'énoncé omniprésent : c'est grâce à vous. Le visionneur réagit, dans la même perspective, pour équilibrer cette politesse qui sera acquittée par la reconnaissance. L'acte de remerciement, à l'origine altruiste, devient, au final, un acte égocentrique implicite. Cela est possible grâce au dispositif qui permet à la fois de mettre l'ego au centre du discours et de mener des interactions tournées sensiblement vers l'autre.

Il est également intéressant de souligner que parfois des vidéos entières sont consacrées aux actes de remerciement (MERCI, SAl4a). La raison du remerciement influe, en effet, sur la place de cet acte : pour remercier le visionnage, l'acte est inséré à la fin des "vidéos », alors que le remerciement du soutien général apparaît plus souvent dans les vidéos "autres ». II convient de remarquer ainsi l'importance des vidéos "autres», qui ont pour finalité d'aménager les faces. Si les youtubeurs s'y engagent, c'est pour atteindre le succès, celui-ci devenant également le moteur du stéréotypage des genres que l'on retrouve sur YouTube. Quoique les vidéos " autres» ne relèvent, au sens strict, ni du podcast, ni du jeu commenté, elles font partie intégrante de l'ethos discursif que le youtubeur représente dans le genre principal.

\section{En guise de conclusion}

Le présent travail avait pour objectif de proposer une analyse qualitative d'actes de langage des youtubeurs les plus célèbres de France afin de nous fournir des pistes pour une analyse quantitative ultérieure. Cette étude a permis de comprendre l'importance de l'argument de l'ethos (vertu partagée) et de celui du pathos (proximité émotionnelle), qui sont tous deux utilisés à des fins de reconnaissance identitaire dans les discours des vidéastes. Dans cette optique, les actes de langage contribuent à construire un ethos à la fois égocentrique et altruiste, arrivant ainsi à définir un ethos quasi-conventionnalisé du métier.

Conformément à cela, l'ouverture et la clôture de la conversation témoignent d'une relation asymétrique tout en indiquant la volonté de prendre en considération l'autre, ce qui crée une proximité avec le destinataire. L'effet de proximité est souvent accentué par des adresses explicites et affectives, qui ont également pour but de capter l'attention des visionneurs. En effet, dans la majorité des cas, le youtubeur accorde un soin tout particulier à l'élaboration de ces actes. Ainsi ces derniers peuvent devenir des actes originaux et récurrents, et se forger parallèlement une 
marque identitaire discursive. Ce fait témoigne du stéréotypage dans la scène générique de YouTube au cœur de laquelle une place importante est accordée aux actes de remerciement qui équilibrent l'asymétrie et renforcent, une fois de plus, la relation parasociale.

Finalement, nous pouvons rapprocher les stratégies discursives des youtubeurs de celles des leaders politiques, qui ont recours à la fois à une logique de proximité et à une logique de distanciation (Donot \& Emediato, 2015, p. 3). En effet, les youtubeurs peuvent être considérés comme les « leaders » identitaires des jeunes de ces dernières années (Baie, 2018 ; Mathy \& Fanouillère, 2019).

\section{Bibliographie}

Amossy, R. (2010). La présentation de soi. Ethos et identité verbale. Paris: Presses Universitaires de France.

Amossy, R. (2012). Argumentation dans le discours. Paris : Armand Colin.

BAIE, F. (2018). Les youtubeurs: impact identitaire et communautaire sur les adolescents ? Analyse UFAPEC, 2. http://www.ufapec.be/nos-analyses/0218youtubeurs.html [20/03/2021].

BARTHA, Cs. (1998). A szociolingvisztika alapjai. Budapest: ELTE. Coll: Magyar Nyelvészeti Továbbképzési Füzetek 2.

CharAUDEAU, P. (2006). Des catégories pour l'humour? Questions de communication, 10, pp. 19-41.

COMbe Celik, C. (2014). Vlogues sur YouTube: un nouveau genre d'interactions multimodales. Premier Colloque IMPEC : Interactions Multimodales Par Ecran. https://impec.sciencesconf.org/conference/impec/pages/Impec2014_Combe _Celik.pdf [06/07/2020].

De Beaugrande, R. \& Dressler, W. (2000). Bevezetés a szövegnyelvészetbe. Budapest : Corvina.

DONOT, M. \& EMEDIATO, W. (2015). La construction de la figure des leaders. Ethos, identité et charisme en perspective comparée. https://doi.org/10.4000/rfsic.1588 [20/03/2021].

FILYO, F. (2021). Diskurzusok a klímaváltozásról: a YouTube és a nemsztenderd nyelvváltozatok a francia nyelvben. Adsumus XIX, pp. 87-111.

GALLANT, N. (2017). Observatoire, Jeunes et société. Bulletin d'information, 14 (2).

GLOzER, R. \& GULD, Á. (2015). Új média - új típusú sztárok? A YouTube magyar hírességei. Információs társadalom, 15 (2), pp. 18-33.

KERBRAT-ORECCHIONI, C. (2014). Les actes de langage dans le discours. Théorie et fonctionnement. Paris : Armand Colin.

MAINGUENEAU, D. (2002). L'ethos, de la rhétorique à l'analyse du discours. http://dominique.maingueneau.pagesperso-orange.fr/pdf/Ethos.pdf [16/10/2020].

MARSHALL, D. P. (2010). The Promotion and Presentation of the Self: Celebrity as a Marker of Presentational Media. Celebrity Studies, 1 (1), pp. 35-48.

MATHY, A. \& FANOUILLERE, J-B. (2019). Énonciation subjective et simulacre d'intimité sur Youtube. Le cas de I'ASMR. Études digitales, 7, Youtoubeurs, youtubeuses : inventions subjectives, pp. 49-62. 
PierozAK, I. (201 1). Les corpus électroniques en sciences du langage : un eldorado ? Le discours et la langue. Revue de linguistique française et d'analyse du discours, 2 (1), pp. 15-31.

RAKOTONOELINA, F. (2011). Analyse du discours, corpus internet et recherche qualitative : objets d'étude et objets de recherche. Le discours et la langue. Revue de linguistique française et d'analyse du discours, 2 (1), pp. 33-53. 